\section{Pregnancy Outcome in Idiopathic Inflammatory Myopathy Patients in a Multicenter Study}

\section{To the Editor:}

Pregnancy in autoimmune diseases may be complicated for both the fetus and the mother. Polymyositis (PM) and dermatomyositis (DM) are autoimmune diseases that affect women more often than men, and can occur before childbearing years ${ }^{1,2}$. Complications during pregnancy were frequently reported in patients with $\mathrm{PM} / \mathrm{DM}$ with active disease, whereas there seems to be a low risk for the fetus and mother in women with a well-controlled disease at time of conception ${ }^{2,3,4,5,6,7,8,9,10}$

Our aims were to assess the pregnancy outcome in myositis. Based on international collaboration, records for women with PM/DM who had been pregnant after the diagnosis of myositis were searched in 4 countries using the clinical databases of contributing hospitals. There were 23 women identified: Czech Republic ( $(=8)$, Hungary $(n=9)$, Sweden $(n=5)$, and Poland $(\mathrm{n}=1)$. All 23 women were white. Medical records were retrospectively reviewed using a standardized protocol.

Normal delivery was defined when a healthy newborn weighing $>2500$ g was delivered after 37 weeks of pregnancy. Premature delivery was defined when the pregnancy ended between gestational weeks 24 and 37. Abortion was defined when the pregnancy ended before week 12 . Intrauterine death was considered a late abortion between weeks 12 and 24.

Disease activity was measured before and during pregnancy, as well as after delivery based on elevation of serum muscle enzymes (creatine kinase and/or lactate dehydrogenase), proximal muscle weakness (using manual muscle strength testing), and presence of a characteristic skin rash in DM. Remission was defined as stable improvement or normalization of muscle strength and muscle enzymes, and the disappearance of skin rashes.

Serological profiles including anti-Jo1, PL-7, PL-12, EJ, OJ, SRP, PM-Scl, Ku, Mi-2, and U1-RNP autoantibodies were measured by ELISA, immunoblot, and immunoprecipitation. We used the Fisher's exact test and the logistic regression test for statistical analysis. The study was approved by the local ethics committees in each country.

In the study population, 10 women had PM, 10 had DM, and 3 had juvenile-onset DM. Among these 23 patients, 3 had an overlap syndrome with rheumatoid arthritis, systemic sclerosis, and systemic lupus erythematosus. One of the patients had antisynthetase syndrome (anti-Jo1 autoantibody positivity, alveolitis, and polyarthritis) before pregnancy, and her muscle weakness appeared after delivery. Clinical data at disease onset are presented in Figure 1. Myositis-specific autoantibodies were present in 10 women as follows: anti-Jo1 $(\mathrm{n}=6)$, anti- $\mathrm{Pm} / \mathrm{Scl}(\mathrm{n}=2)$, and anti-Mi-2 $(\mathrm{n}=2)$. Other autoantibodies were also detected, such as anti-SSB $(\mathrm{n}=1)$, antihiston ( $\mathrm{n}=1)$, anti-DNA $(\mathrm{n}=2)$, and anticardiolipin $(\mathrm{n}=5)$.

There were 33 pregnancies recorded in the 23 women after or at the time of onset of PM/DM (Table 1). We found 3 cases of pregnancy-induced myositis. Nineteen pregnancies ended in delivery of a healthy baby, without complications. Twelve of these pregnancies were conceived in remission. We used a low-dose corticosteroid treatment $(8-12 \mathrm{mg})$ or no medication during pregnancy. Two patients with PM had flares after delivery; they needed methotrexate (MTX), azathioprine, and cyclosporine (CSA) treatment (Table 1). Six pregnancies were recognized during the active phase of the mother's disease, but the elevation of corticosteroid dosage was effective until delivery (mean dose was $24 \mathrm{mg} /$ day), after which were started MTX, CSA, or chloroquine (Table 1). Mean duration of healthy pregnancy was 39.6 weeks (37-41 weeks) in cases conceived in remission and 37.5 weeks (37-38 weeks) in women with active disease. The mean weight of newborns was $2698 \mathrm{~g}(1680-3220 \mathrm{~g})$ in women with

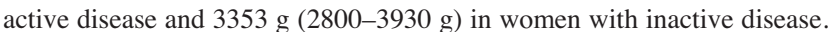

We found complications in 14 pregnancies. These pregnancies ended between gestational weeks 7 and 31 . Three pregnancies ended with intrauterine death, 6 with abortion (4 spontaneous and 2 induced), and 1 with extrauterine pregnancy. Four babies were born prematurely. Mean birth weight was $2350 \mathrm{~g}(1680-3220 \mathrm{~g})$, and the delivery happened at gestational week 35.75 (35-36 weeks). To the best of our knowledge, these babies developed normally thereafter. The indications of induced abortions were the mother's serious disease activity or fetal malformation. In those cases, the mother needed immunosuppressant treatment. These 14 complicated pregnancies had been observed in 9 women $(\mathrm{PM}=6, \mathrm{DM}=3)$. Three women had anti-Jo1 positivity, and anticardiolipin or anti- $\mathrm{Pm} / \mathrm{Scl}$ autoantibodies were also detected in these cases. Seven women $(\mathrm{PM}=5, \mathrm{DM}$ = 2) needed immunosuppressive treatment before, during, or after pregnancy (Table 1). Two of these complicated cases were pregnancy-induced. One ended with spontaneous abortion and one with premature delivery; both mothers had persistent disease activity after delivery.

In our study, no severe maternal complications were reported. The fetal complications associated more frequently with PM than with DM $(\mathrm{p}=$ $0.0729)$. The relative risk of complicated pregnancy in PM is $1.923(\mathrm{CI}$ 95\% 1041-3553). Besides the PM subset, the disease activity before or

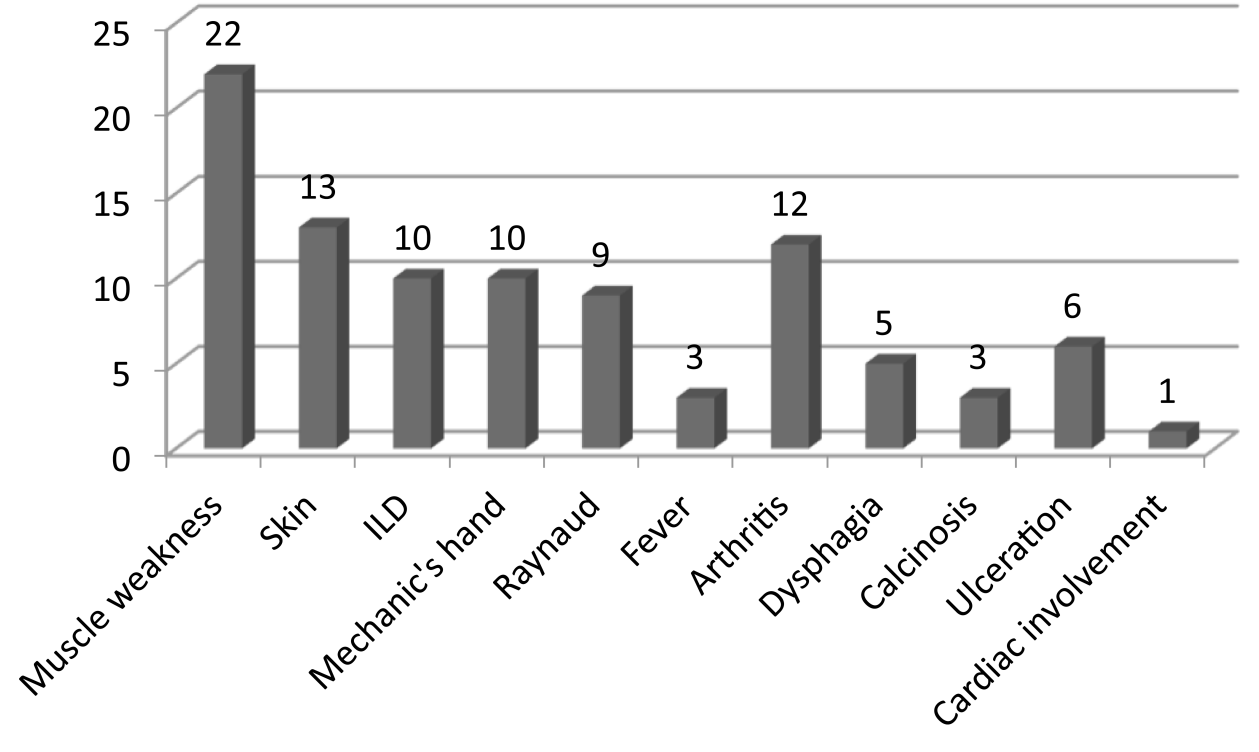

Figure 1. Clinical data at disease onset. Numbers indicate number of women with each condition. "Skin" refers to skin rashes 
Table 1. Main data about the pregnancies.

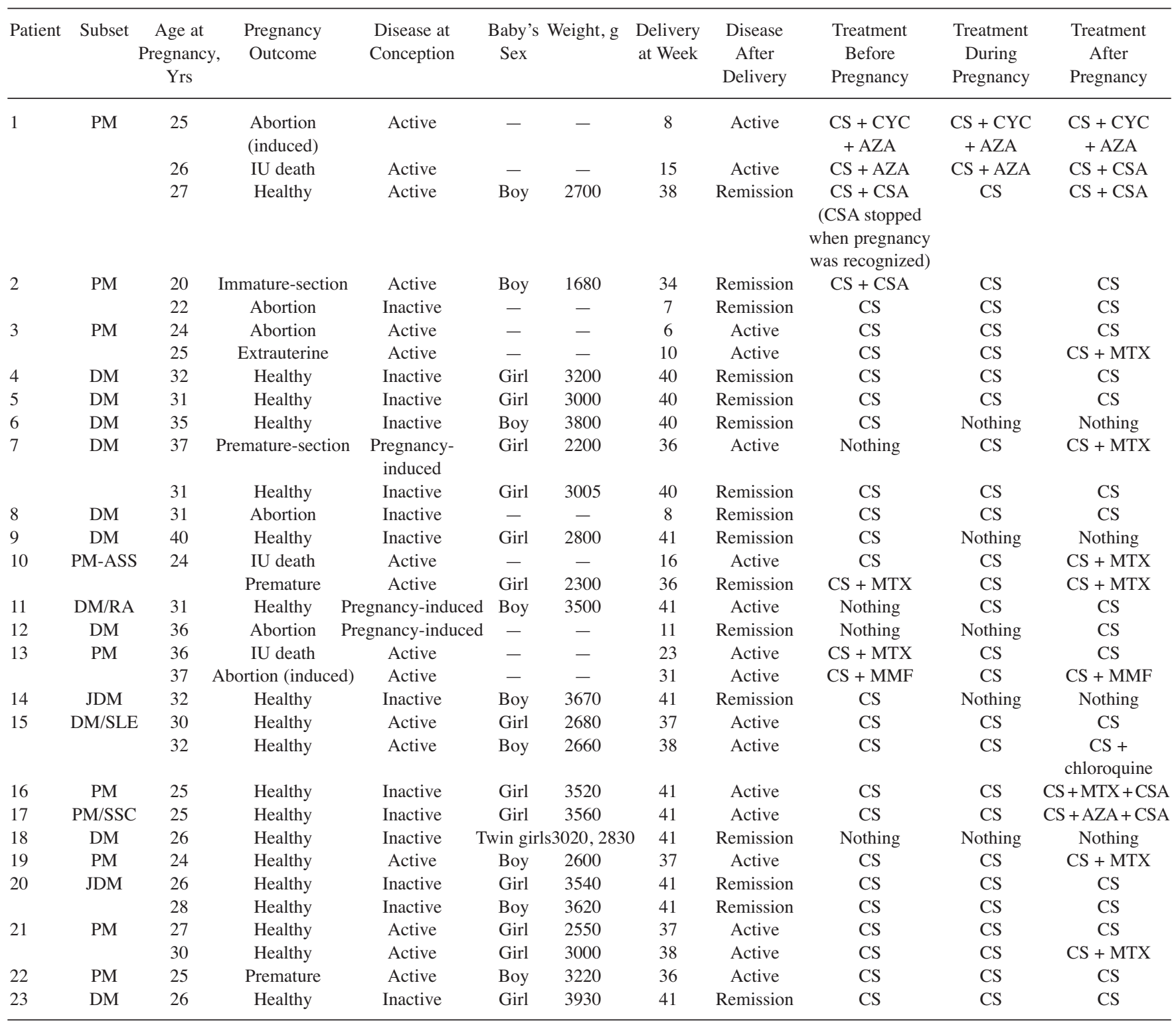

PM: polymyositis; DM: dermatomyositis; ASS: antisynthetase syndrome; RA: rheumatoid arthritis; JDM: juvenile dermatomyositis; SSC: systemic sclerosis; SLE: systemic lupus erythematosus; IU: intrauterine; CS: corticosteroid; CYC: cyclophosphamide; AZA: azathioprine; CSA: cyclosporine; MTX: methotrexate; MMF: mycophenolate mofetil.

during the pregnancy and the presence of autoantibodies seem to be risk factors for complications. Logistic regression analysis proved that high pregnancy risk is associated with joint involvement (OR 7.5, $\mathrm{p}=0.032$, $95 \%$ CI $1.19-47.77)$ and anti-Jo1 positivity (OR 8.9, $\mathrm{p}=0.023,95 \%$ CI $1.34-58.88)$

\section{ACKNOWLEDGMENT}

This study was organized within the ESF EuMyoNet Research Networking Programme. We thank Andrea Váncsa from the Division of Clinical Immunology, Department of Internal Medicine, University of Debrecen, Hungary; Lenka Plestilova from the Institute of Rheumatology and Clinics of Rheumatology First Faculty of Medicine, Charles University, Prague, Czech Republic; Karina R. Gheorghe from the Rheumatology Unit, Department of Medicine, Karolinska University Hospital, Solna, and the
Karolinska Institutet, Stockholm, Sweden; Balsam Hanna from the Sahlgrenska University Hospital, Gothenburg, Sweden; Ewa Wiesik-Szewczyk from the Institute of Rheumatology, Warsaw, Poland; and Peter J. Charles from the Kennedy Institute, London, UK.

MELINDA NAGY-VINCZE, MD, Division of Clinical Immunology, Department of Internal Medicine, University of Debrecen, Hungary; JIRI VENCOVSKY, MD, PhD, Institute of Rheumatology and Clinics of Rheumatology First Faculty of Medicine, Charles University, Prague, Czech Republic; INGRID E. LUNDBERG, MD, PhD, Rheumatology Unit, Department of Medicine, Karolinska University Hospital, Solna, and the Karolinska Institutet, Stockholm, Sweden; KATALIN DANKÓ, MD, PhD, Division of Clinical Immunology, Department of Internal Medicine, University of Debrecen, Hungary. Address correspondence to Dr. M. 
Nagy-Vincze, Division of Clinical Immunology, Department of Internal Medicine, University of Debrecen, Moricz Zs. u. 22, H-4032 Debrecen, Hungary. E-mail: melinda.nagyvincze@gmail.com

\section{REFERENCES}

1. Nelson JL. Pregnancy immunology and autoimmune disease. J Reprod Med 1998;43:335-40.

2. Váncsa A, Ponyi A, Constantin T, Zeher M, Dankó K. Pregnancy outcome in idiopathic inflammatory myopathy. Rheumatol Int 2007;27:435-9.

3. Williams L, Chang PY, Park E, Gorson KC, Bayer-Zwirello L. Successful treatment of dermatomyositis during pregnancy with intravenous immunoglobulin monotherapy. Obstet Gynecol 2007;109:561-3.

4. Kanoh H, Izumi T, Seishima M, Nojiri M, Ichiki Y, Kitajima Y. A case of dermatomyositis that developed after delivery: the involvement of pregnancy in the induction of dermatomyositis. $\mathrm{Br}$ J Dermatol 1999;141:897-900.

5. Behan WM, Behan PO. Recent advances in polymyositis. Ital $\mathbf{J}$ Neurol Sci 1984;5:23-31.
6. Suwa A, Hirakata M, Tsuzaka K, Hama N, Fujii T, Mimori T, et al. [Spontaneous remission of dermatomyositis which developed one month after normal delivery]. [Article in Japanese] Ryumachi 1992;32:73-9.

7. Park IW, Suh YJ, Han JH, Shin YS, Choi JH, Park HS, et al. Dermatomyositis developing in the first trimester of pregnancy. Korean J Intern Med 2003;18:196-8.

8. Silva CA, Sultan SM, Isenberg DA. Pregnancy outcome in adult-onset idiopathic inflammatory myopathy. Rheumatology 2003;42:1168-72.

9. Messina S, Fagiolari G, Lamperti C, Cavaletti G, Prelle A, Scarlato $\mathrm{G}$, et al. Women with pregnancy-related polymyositis and high serum CK levels in the newborn. Neurology 2002;58:482-4.

10. Rosenzweig BA, Rotmensch S, Binette SP, Phillippe M. Primary idiopathic polymyositis and dermatomyositis complicating pregnancy: diagnosis and management. Obstet Gynecol Surv 1989;44:162-70.

J Rheumatol 2014;41:12; doi:10.3899/jrheum.140438 